\title{
Development and feasibility testing of an oral hygiene intervention for stroke unit care
}

DOI:

10.1111/ger.12232

\section{Document Version}

Accepted author manuscript

Link to publication record in Manchester Research Explorer

\section{Citation for published version (APA):}

Smith, C. J., Horne, M., McCracken, G., Young, D., Clements, I., Hulme, S., Ardron, C., Hamdy, S., Vail, A., Walls, A., \& Tyrrell, P. (2016). Development and feasibility testing of an oral hygiene intervention for stroke unit care. Gerodontology. https://doi.org/10.1111/ger.12232

\section{Published in:}

Gerodontology

\section{Citing this paper}

Please note that where the full-text provided on Manchester Research Explorer is the Author Accepted Manuscript or Proof version this may differ from the final Published version. If citing, it is advised that you check and use the publisher's definitive version.

\section{General rights}

Copyright and moral rights for the publications made accessible in the Research Explorer are retained by the authors and/or other copyright owners and it is a condition of accessing publications that users recognise and abide by the legal requirements associated with these rights.

\section{Takedown policy}

If you believe that this document breaches copyright please refer to the University of Manchester's Takedown Procedures [http://man.ac.uk/04Y6Bo] or contact uml.scholarlycommunications@manchester.ac.uk providing relevant details, so we can investigate your claim.

\section{OPEN ACCESS}




\title{
Development and feasibility testing of an oral hygiene intervention for stroke unit care
}

\author{
Craig J. Smith ${ }^{1,2}$, Maria Horne ${ }^{3}$, Giles McCracken ${ }^{4}$, David Young ${ }^{5}$, Ian Clements ${ }^{6}$, \\ Sharon Hulme ${ }^{2}$, Claire Ardron ${ }^{1}$, Shaheen Hamdy ${ }^{7}$, Andy Vail ${ }^{8}$, Angus Walls ${ }^{9}$ and \\ Pippa J. Tyrrell ${ }^{1,2}$
}

${ }^{1}$ Greater Manchester Comprehensive Stroke Centre, Manchester Academic Health Science Centre, Salford Royal Foundation Trust, Salford, UK; ${ }^{2}$ Stroke and Vascular Centre, Institute of Cardiovascular Sciences, University of Manchester, Manchester, UK; ${ }^{3}$ Faculty of Health Studies, School of Nursing, University of Bradford, Bradford, UK; ${ }^{4}$ School of Dental Sciences, Newcastle University, Newcastle, UK; ${ }^{5}$ The Greater Manchester School for Dental Care Professionals (MANDCP), Salford, UK; ${ }^{6}$ Patient, Carer and Public Involvement Group, North West Stroke Research Network, Salford Royal NHS Foundation Trust, Salford, UK; ${ }^{7}$ Centre for Gastrointestinal Sciences, Institute of Inflammation and Repair, University of Manchester, Manchester, UK; ${ }^{8}$ Centre for Biostatistics, Institute of Population Health, University of Manchester, Manchester, UK; ${ }^{9}$ Edinburgh Dental Institute, University of Edinburgh, Edinburgh, UK

Gerodontology 2016; doi:10.1111/ger.12232

\section{Development and feasibility testing of an oral hygiene intervention for stroke unit care}

Objective: To develop an oral hygiene complex intervention and evaluate its feasibility in a single UK stroke centre.

Background: Oral hygiene interventions might improve clinical outcomes after stroke but evidencebased practice is lacking.

Materials and methods: We used a sequential mixed methods approach and developed an oral hygiene complex intervention comprising: (i) web-based education and 'hands-on' practical training for stroke unit nursing staff, (ii) a pragmatic oral hygiene protocol consisting of twice-daily powered (or manual if preferred) brushing with chlorhexidine gel (or non-foaming toothpaste) \pm denture care. We evaluated feasibility of (i) the staff education and training and (ii) the oral hygiene protocol in consenting inpatients with confirmed stroke, requiring assistance with at least one aspect of personal care.

Results: The staff education and training were feasible, acceptable and raised knowledge and awareness. Several barriers to completing the education and training were identified. The oral hygiene protocol was feasible and well-tolerated. $22 \%$ of eligible patients screened declined participation in the study. Twenty-nine patients (median age $=78$ year; National Institutes of Health Stroke Scale score $=8.5 ; 73 \%$ dentate) were recruited at a median of 7 days from stroke onset. $97 \%$ of participants chose the default chlorhexidine-based protocol; the remainder chose the non-foaming toothpaste-based protocol. The mouth hygiene protocol was administered as prescribed on $95 \%$ of occasions, over a median duration of 28 days. There were no adverse events attributed to the oral hygiene protocol.

Conclusion: Our oral hygiene complex intervention was feasible in a single UK stroke centre. Further studies to optimise patient selection, model health economics and explore efficacy are now required.

Keywords: oral hygiene, complex intervention, stroke, education.

Accepted 4 April 2016

\section{Introduction}

Stroke is the second most common cause of death worldwide $^{1}$ and the leading cause of adult complex neurological disability ${ }^{2}$. Around 152000 people in the UK have a stroke annually and approximately $25 \%$ die within the first year ${ }^{3}$. A growing body of evidence suggests a link between stroke and dental disease. Periodontal disease is associated with progression of atherosclerosis ${ }^{4,5}$ and incident cerebrovascular disease ${ }^{6}$. Patients with stroke have a higher prevalence of periodontal disease, tooth loss and removable dentures than non-stroke controls and are less likely to visit a dentist annually ${ }^{7}$.

Following stroke, poor oral health may also contribute to worse clinical outcomes, by increasing the risk of aspiration pneumonia ${ }^{8}$, affecting quality 
of life and impacting on nutrition ${ }^{9}$. Pneumonia remains a serious and frequent complication of stroke, particularly in older individuals with swallowing problems, and there are currently very limited preventative strategies available. Clinical trials of oral hygiene interventions have been reported to reduce the incidence of pneumonia in other clinical settings, such as the intensive care unit and in hospitalised or institutionalised older people ${ }^{10,11}$. However, provision of oral care for hospitalised stroke patients is a neglected yet challenging area of stroke care. Delivering oral hygiene to stroke patients poses appreciable challenges due to the frequent occurrence of physical, communication and cognitive impairments ${ }^{12}$, yet is important to stroke patients ${ }^{13}$. Provision of oral hygiene, including cleaning of the oral cavity, teeth and dentures, and availability of oral care equipment, varies considerably between stroke units ${ }^{13,14}$. Oral hygiene in UK stroke units is provided or supervised by both trained nursing staff and healthcare assistants (HCAs), but specific protocols or training are lacking, and staff felt they had insufficient knowledge and training to deliver oral hygiene effectively ${ }^{13}$.

Several early-phase studies have evaluated oral hygiene interventions in hospitalised stroke patients, but have differed considerably in the populations studied (ventilated vs. non-ventilated patients), timing from stroke onset, healthcare environment (intensive care, acute stroke unit, rehabilitation unit), interventions used and outcome measures ${ }^{15-19}$. At present, there remains a lack of evidence-based guidance informing optimal education and training for professionals delivering oral care after stroke, or oral hygiene protocols for stroke unit care. Our overall aim was therefore to develop an oral hygiene intervention, incorporating both staff education and training and an oral hygiene protocol and evaluate its feasibility in a UK hospital stroke service. Our objectives were to:

1. Formulate a transferrable education and training programme for stroke unit nursing staff and evaluate feasibility, acceptability and adequacy.

2. Develop a pragmatic and comprehensive oral hygiene protocol for stroke unit care and evaluate feasibility, safety and acceptability.

\section{Materials and methods}

We used a sequential mixed methods approach ${ }^{20}$ and report our methodology and findings in line with CReDECI 2 guidelines for the development and evaluation of complex interventions ${ }^{21}$. The study took place at the Greater Manchester
Comprehensive Stroke Centre (CSC), Salford Royal NHS Foundation Trust (SRFT), involving both the acute stroke unit (ASU) and stroke rehabilitation unit (SRU). A flow chart outlining the overall study design is shown in Fig. 1. The study was approved by the National Research Ethics Service, North West Committee (REC reference 13/NW/0130) and University of Manchester.

\section{Stage 1: rationale, development and description of the intervention}

We developed our complex intervention in line with MRC guidance ${ }^{22}$, considering the evidence from an existing systematic review in the field ${ }^{23}$, the rationale for the intervention and the requirement to evaluate implementation of the intervention in the clinical care setting.

Oral hygiene protocol. The main rationale and requirements were to comprehensively clean the oral cavity ( \pm dentures), whilst considering particular challenges of oral care in stroke patients, existing variation in oral care practices and safety (e.g. potential aspiration risk). In the absence of an evidence-based oral hygiene protocol for stroke unit care, the final study protocol, reached by consensus amongst the multidisciplinary study team and service user engagement, is presented in Table 1. Powered brushing was chosen as the default method as it is more effective at reducing plaque and gingivitis than manual brushing in participants without disabilities affecting their oral care $^{24}$. Because of potential concerns around aspiration during brushing, the options in terms of cleansing agent were either a non-foaming toothpaste or chlorhexidine gel. To enhance the likelihood of reducing oral bioburden, we chose a chemically active antimicrobial agent (chlorhexidine) as the default cleansing agent rather than a conventional non-foaming toothpaste. Providing mechanical oral hygiene is performed well, there is good evidence that a paste per se is not required for cleaning, but the additional benefit of chemical inhibition of oral microflora is likely beneficial in this circumstance ${ }^{25}$. The default protocol therefore incorporated powered brushing (unless completely edentulous) with chlorhexidine gel, with the option for participants to initially choose or switch to manual brushing or non-foaming toothpaste. This was to allow some flexibility and therefore maximise participation and retention. The powered toothbrush used in the protocol was a Philips Sonicare FlexCare+ (HX6942/20). Bedside suction during administration of brushing 
Figure 1 Flow chart outlining overall study design.

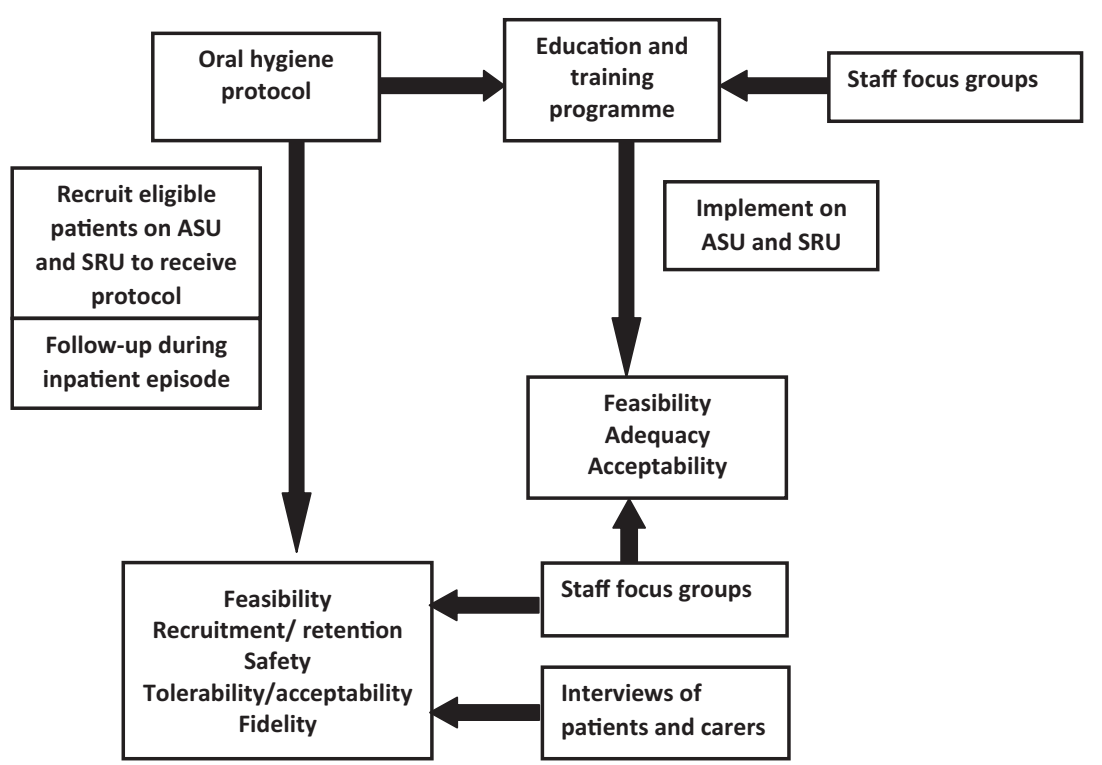

was encouraged at the discretion of the ward staff. Additional oral care was permitted, provided toothpaste was avoided for at least 1 hour after chlorhexidine administration.

Staff education and training. Limited randomised trial evidence supports education and training for staff delivering mouth hygiene ${ }^{15,26}$, although the optimal content, format and assessment methods have yet to be determined. We therefore first undertook an exploratory, qualitative approach using focus groups of healthcare professionals who worked on the ASU and SRU, using open-ended questions to identify the education and training needs. Two focus groups were conducted between March and July 2012 with a purposive sample of 6-8 stroke unit staff $(n=10$ in total) to identify and inform the education and training needs of stroke nurses and HCAs to deliver the mouth hygiene protocol. This phase fed directly into the development of the content, format and competencies of the education and training programme. A 1-day multidisciplinary workshop was undertaken to develop the content and format of the training and competency assessments and the logistics of delivering this. A web-based resource was favoured for the core content and competency assessments, in view of the logistical problem of releasing multiple staff for classroom-based teaching sessions. This also provided the advantage of a transferable resource across sites for any subsequent multicentre study. The webbased resource was developed in collaboration with an independent, external web-design service (www.eibs.co.uk). The final content, competencies and format of the education and training were reached by discussion and consensus within the study team. The web-based resource covered 'core taught material', comprising four discrete modules, each with learning objectives and self-assessment questions required to be successfully completed before moving to the next module. The modules covered an introduction, anatomy and physiology, common dental problems on assessment (Fig. 2a and b) and video clips demonstrating a dental hygienist administering the study oral hygiene care protocols (powered and manual brushing, plus denture brushing and care) to a stroke survivor.

In addition, three staff each from the ASU and SRU (designated 'oral care link nurses') received additional education and training at MANDCP (The Greater Manchester School for Dental Care Professionals), to facilitate 'hands-on' training of the stroke unit staff on the ward setting once they had completed the web-based programme.

\section{Stage 2: evaluation of the intervention}

Staff education and training. The web-based education and training was launched on 8 April 2013 and implemented across both ASU and SRU. Nursing staff and HCAs accessed the website using individualised login details, and the stroke services matron was automatically emailed when a staff member accessed the site, and whether the module was completed successfully or not. Nursing staff and HCAs were assessed as competent in the practical elements of the oral hygiene protocol by 
Table 1 Description of the oral hygiene protocol.

Cleaning teeth and the oro-mucosal surfaces

Morning (after breakfast for those taking oral nutrition) and evening

After preparing the clinical environment appropriately, the procedure will be carefully explained to the patient and/or carer.

Position participants seated upright or semirecumbent if bed-bound. Bedside suction can be used concurrently if aspiration is considered likely. Wear disposable, protective visors, aprons and gloves.

Clean the teeth using a powered toothbrush and chlorhexidine gel $(1 \%)^{a}$ for 2 mins followed by tongue surface cleaning using a mechanical tongue scraper and chlorhexidine gel (1\%). A manual toothbrush or non-foaming toothpaste can be used as alternatives either from study entry or at any stage should the participant prefer (and the prescription chart updated).

Completely edentulous patients can still receive the protocol, using the brush to gently apply the chlorhexidine or toothpaste.

Clean the remaining oro-mucosal surfaces with chlorhexidine gel (1\%) applied with a sponge applicator.

Confirm protocol administered by signing the prescription chart and record if not administered and reasons why.

Dry or cracked lips should be cleaned with moistened gauze and protected with a lubricant (simple petroleum jelly). Oral lubrication may be offered, for example moistened gauze, artificial saliva substitutes.

Other aspects of usual mouth care may also be delivered at the discretion of the nursing staff(but more than $\mathrm{l} \mathrm{h}$ before or after the chlorhexidine intervention if using toothpaste).

Denture care

Evening

Dentures will be labelled with the owner's name and scrubbed clean daily with non-perfumed soap and water using a denture brush, rinsed in cold water then immersed in Milton (all plastic dentures) or chlorhexidine solution (those with a metal framework) in sealed denture pots overnight. Other denture care will be at the discretion of individual participants/staff.

${ }^{a}$ Or an alternative if known to have an allergy to chlorhexidine.

one of the six link nurses in small-group teaching sessions using a dental simulator.

Outcome measures: Feasibility was assessed as the time taken for all nursing staff and HCAs on the ASU and SRU to successfully complete the web-based training (using linkage to individual staff login and module completion) and to be (a)

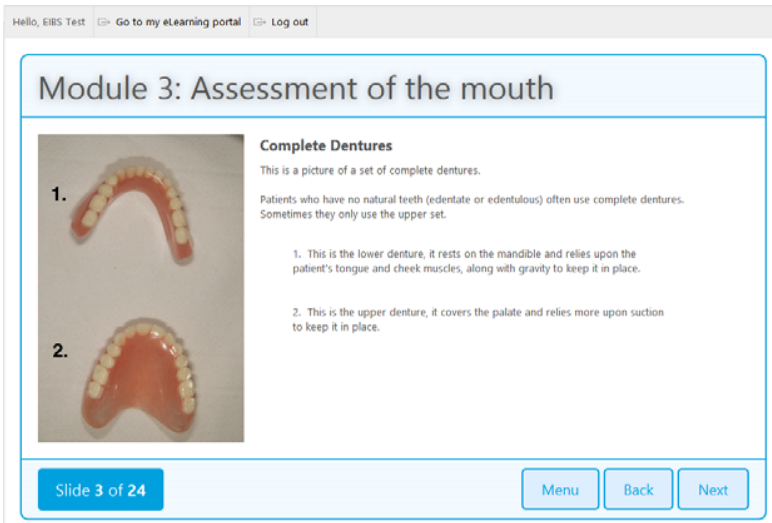

(b)

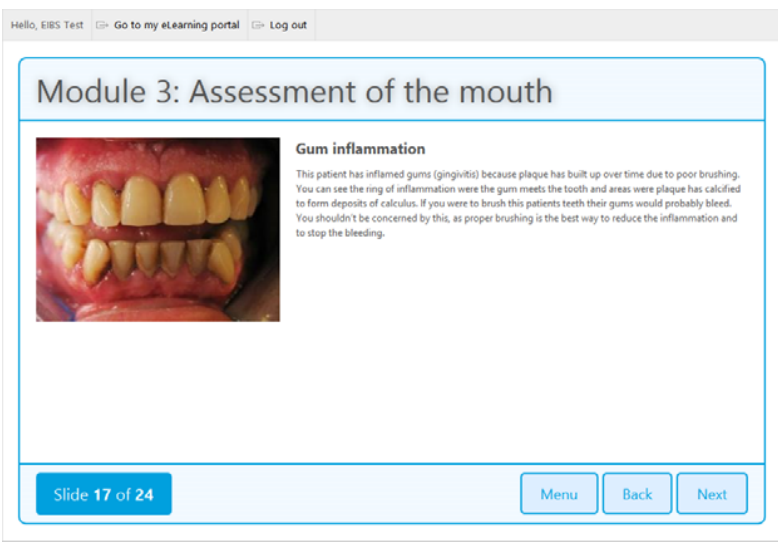

Figure 2 Example screenshots of the web-based education and training resource. $(a, b)$ Assessment of the mouth.

registered as competent following the 'hands-on' practical sessions.

Adequacy and acceptability were assessed in three subsequent, additional focus groups of ASU and SRU staff ( $n=13$ total), purposively sampled to reflect experience and skill mix. These were undertaken after the education and training programme had been implemented, and the evaluation of the oral care protocol was underway.

Oral hygiene protocol. Patients: Patients with confirmed stroke on the ASU or SRU, expected to remain as inpatients at SRFT $>72 \mathrm{~h}$, and requiring assistance with at least one aspect of personal care, were invited to participate and receive the oral hygiene protocol until discharge from inpatient stroke services at SRFT. The CSC at SRFT operates a hub and spoke model, and patients residing outside the local SRFT catchment who present to the CSC in the hyperacute or acute phase are repatriated to their base hospital after completion of an acute care bundle. Therefore, 
only patients with a Salford postcode were considered for screening (to avoid early repatriation of participants to their base hospital). Patients with rapidly improving symptoms, receiving end of life care or concurrent treatment for pneumonia at the time of screening were excluded. Patients with a known allergy to chlorhexidine were not excluded, but were offered the chlorhexidine-free non-foaming toothpaste if otherwise willing to participate. Patients were screened for participation as soon as possible after admission. A log was kept of all patients screened for the study, including age, sex and the reason for non-inclusion. Consent was provided by all participating patients or by a personal consultee in situations where capacity was lacking.

Study procedure: Baseline assessment included pre-stroke independence [modified Rankin Scale (mRS)], past medical history, current medications, smoking status, alcohol history, characteristics of presenting stroke [date and time of onset, hemisphere affected, stroke subtype, stroke severity using National Institutes of Health Stroke Scale (NIHSS)], swallow and nutrition status (nil by mouth, modified diet or fluids, tube feeding). Baseline oral assessment using The Holistic and Reliable Oral Assessment Tool (THROAT) ${ }^{27}$ was documented by a member of the research nursing team. Participating patients received the study oral hygiene protocol (Table 1) as part of their routine nursing care until the end of the study period, or discharge from inpatient stroke services at SRFT. The oral hygiene protocol was prescribed on the medication chart, administered as per protocol by the clinical nursing staff and signed for by a trained nurse to facilitate recording of fidelity of administration. Staff were encouraged to record reasons for non-receipt of the protocol. If participants chose to switch from chlorhexidine to non-foaming toothpaste or from powered to the manual brush regimen, the protocol was re-prescribed accordingly. The ability of patients to administer their own mouth or denture care using the study protocol, or for their carers to provide the care, was considered regularly in individual circumstances following multidisciplinary assessment. Relevant instruction and supervision was provided by the nursing staff and HCAs.

Outcome measures: Feasibility was evaluated as follows: proportion potentially eligible from screening log, proportion of those eligible declining participation and reasons given; fidelity, tolerability, acceptability: proportion of participants unable to receive the protocol on one or more occasions and reasons given, proportion of prescribed doses actually received for each participant, proportion of participants switching to manual brush/non-foaming toothpaste and reasons given; number of withdrawals from study and reasons given; safety: adverse events including episodes of pneumonia (defined by clinician-initiated antibiotic therapy). In addition, exploratory data on patient outcomes were also recorded including length of stay, end of study mRS and survival (incorporating a score of six for death on the mRS).

As this was a feasibility study, no power calculation was performed. A sample size of 30 inpatients with stroke recruited over the 5-month period (including follow-up) for which funding was available was estimated based on preceding admissions data. Up to 10 semi-structured interviews of patients and/or their carers were undertaken to assess tolerability and acceptability, which was anticipated to reach saturation.

Data analysis. All focus groups and interviews were undertaken by an experienced qualitative researcher $(\mathrm{MH})$. Focus groups and interviews were audio-recorded, transcribed verbatim and conducted until data saturation was reached. Verbatim transcripts were anonymised and pseudonyms used. Data were analysed using framework approach ${ }^{28}$. MH coded all transcripts, using NVIVO10 qualitative computer package. Codes were then discussed with the research team to develop an agreed indexing scheme to chart data. Charts were then shared with the research team to explore and interpret the data together and agree on the final themes. Consensus was reached through discussion. Quantitative data were presented using appropriate summary statistics, using SPSS version 22 .

\section{Results}

\section{Evaluation of the staff education and training programme}

Feasibility. The web-based education went live on 8 April 2013, and the six ASU or SRU link nurses underwent additional training at MANDCP on 10 May 2013. The web-based education and training and the 'hands-on' practical competencies were completed by all 50 nursing staff and HCAs within a 2 -month period (54 days). This incorporated staff annual and sick leave, weekends and those on permanent shift patterns (e.g. staff on permanent nights).

Acceptability, barriers and adequacy. In total, three focus groups with 3-5 stroke unit staff $(n=13$ in 
total) were conducted after the implementation of the education and training programme. The participants were HCAs, staff nurses or ward sisters, with between 4 months and 6 years of experience of working in hospital stroke care. Three main themes emerged: acceptability, barriers and adequacy. A summary of these findings, with illustrative verbatim quotes to support these themes, is presented in Online Only Table S1.

Acceptability: Most participants found the webbased education and training programme acceptable and easy to use. A few would have liked to have some supplementary classroom-based teaching. Although most found the simulation exercise very useful, some felt that it was not 'real life' in that difficulties encountered on 'real' people were dissimilar.

Barriers: Several barriers to completing the training and education were identified. Access was an issue for some staff; this was mainly around logging onto the online system. Getting interrupted by ward-related issues, whilst undertaking the educational resource at work, was another reported barrier.

Adequacy: The web-based education materials were reported to be informative, widened healthcare professionals' knowledge of the anatomy of the mouth and assisted them to undertake the mouth hygiene protocol using a dental simulator. The education and training programme also raised healthcare professionals' awareness of the need for mouth care, and the simulation exercise was reported to be particularly useful in building healthcare professionals' self-confidence in their own ability to deliver the oral hygiene protocol in the ward environment.

\section{Evaluation of the oral hygiene protocol}

Screening and recruitment. During the end of April 2013 to March 2014, 945 confirmed strokes were admitted to the CSC at SRFT (Sentinel Stroke National Audit Programme data), of which 293 $(31 \%)$ were from the SRFT catchment area. During the study recruitment period (3 June 2013 to 19 October 2013), we screened 87 patients within the SRFT catchment area, of whom 50 were eligible (Fig. 3). Of these, 11 patients $(22 \%)$ declined participation in the study, mainly due to lack of interest in the study or research participation in general. The baseline characteristics of the 29 participating patients are shown in Table 2. The majority presented with total or partial anterior circulation syndromes, were dentate and were independent (including with their oral care) prior to the index stroke. Participants were recruited at a median (minimum, maximum) of 7 days $(1,55)$ from admission, mainly from the ASU $(59 \%)$. The median (minimum, maximum) duration of participation in the study was 28 days $(7,105)$.

At study entry, the majority of participants $(79 \%)$ required at least assistance with their oral care, and the remainder were fully dependent. Twenty-one $(72 \%)$ agreed to the default powered brush-chlorhexidine gel protocol, 7 (24\%) chose the manual brush-chlorhexidine gel protocol and the remaining participant chose the manual brush-toothpaste protocol. Three of the participants using the powered brush-chlorhexidine gel protocol (14\%) switched to the manual brush within the first 4 days of participation, due to personal preference.

Fidelity and tolerability. When considering all participating patients, the median (minimum, maximum) proportion of all prescribed doses actually received was $95(67,100) \%$, although the majority of participants $(72 \%)$ did not receive the protocol as prescribed on at least one occasion. The main reason for non-receipt of the protocol was participant refusal $(51 \%)$, but was not clearly documented in $35 \%$. Other reasons for non-receipt of the protocol were the following: clinical deterioration $(6 \%)$, concern regarding aspiration risk in enteric-fed patient $(2 \%)$, participant asleep or offward $(2 \%)$, brush not available $(1 \%)$, new member of staff not trained $(1 \%)$, missing data/other $(4 \%)$. The denture protocol was completed as prescribed in all cases. None of the participating patients withdrew consent. The oral hygiene protocol was discontinued in three participants after they deteriorated and commenced palliative care. None of the other participating patients discontinued the protocol.

Safety and clinical outcomes. There were 10 episodes of clinician-diagnosed pneumonia requiring antibiotic treatment, occurring in six participants $(21 \%)$. One participant (baseline NIHSS $=9$, enteric-feeding, history of chronic lung disease) had four discrete episodes of recurrent lower respiratory tract infection. Other adverse events recorded during follow-up included urinary tract infection in $5(17 \%)$, deep vein thrombosis and pulmonary embolism in $2(7 \%)$ and one occurrence each of gastrostomy site infection, paroxysmal atrial fibrillation, acute parotitis, acute pseudogout, acute duodenitis, Clostridium difficile diarrhoea, fall with head and spinal injury. There were no allergies to chlorhexidine recorded. The 
Figure 3 Flow chart showing eligibility and recruitment of patients for oral hygiene protocol feasibility study.

median mRS (minimum, maximum) at completion of follow-up was $4(0,6)$. Three participants $(10 \%)$ died during study follow-up; cause of death was pneumonia in two and ischaemic stroke in the other. None of the episodes of pneumonia or other adverse events were attributed to the oral hygiene protocol.

Acceptability and patient experience. In total, eight interviews were undertaken in eight patients (Online Only Table S2) and three carers during the oral hygiene protocol study period. Four main themes emerged: acceptability, difficulties, benefits and improvements. Online Only Table S3 summarises these findings and provides illustrative quotes to support these themes.

Acceptability: Self-caring participants reported good satisfaction and tolerability with no problems with the oral hygiene protocol and that it left their mouth feeling really clean. Those who needed assistance with mouth care reported that they felt that this was an important part of care. An unexpected finding was that for some patients, the fact that there was an oral hygiene protocol acted as an aide memoir for them to undertake this important aspect of care. The ease and manoeuvrability of the powered toothbrush enabled some patients who had a stroke affecting their dominant hand to self-care in terms of oral hygiene.

Difficulties: The only difficulty reported was around the strength and power of the powered brush. For those who were not self-caring with oral hygiene, lack of privacy when the healthcare professional or carer was undertaking mouth care for them was highlighted as an issue.

Benefits: An unexpected finding of the study was that participating patients reported the added benefit of thinking about their overall health and well-being. Carers who undertook oral hygiene felt that mouth care was an important part of ward-based health care, in order to provide a fresher feel to the patient's mouth. Other carers recognised the importance of oral hygiene to avoid infections that might complicate recovery.

Improvements: In terms of improvement, the only issue raised by one participating patient was around healthcare professionals highlighting the reason for maintaining good mouth care post-stroke. 
Table 2 Baseline characteristics in the oral hygiene protocol feasibility evaluation.

\begin{tabular}{|c|c|}
\hline Median age (minimum, maximum) $(y)$ & $78(48,94$ \\
\hline Male sex $(n, \%)$ & $17(59)$ \\
\hline \multicolumn{2}{|l|}{ Stroke subtype $(n, \%)$} \\
\hline Ischaemic stroke & $29(100)$ \\
\hline \multicolumn{2}{|l|}{ Affected hemisphere $(n, \%)$} \\
\hline Left & $10(35)$ \\
\hline Right & $18(62)$ \\
\hline Bilateral & $1(3)$ \\
\hline \multicolumn{2}{|c|}{ Median NIHSS (minimum, maximum) score } \\
\hline Admission & $8.5(1,26)$ \\
\hline Study entry & $7(1,26)$ \\
\hline \multicolumn{2}{|c|}{ Stroke syndrome classification (OCSP) $(n, \%)$} \\
\hline TACI & $11(38)$ \\
\hline PACI & $7(24)$ \\
\hline POCI & $6(21)$ \\
\hline LACI & $5(17)$ \\
\hline Nil by mouth $(n, \%)$ & $11(38)$ \\
\hline \multicolumn{2}{|l|}{ Nutrition $(n, \%)$} \\
\hline Normal & $13(45)$ \\
\hline Modified diet/fluids & $5(17)$ \\
\hline Tube feeding & $11(38)$ \\
\hline Dentate (at least one tooth) $(n, \%)$ & $21(73)$ \\
\hline Denture wearers $(n, \%)$ & $15(52)$ \\
\hline \multicolumn{2}{|l|}{ Usual mouth care } \\
\hline Independent & $25(86)$ \\
\hline Requires assistance & $4(14)$ \\
\hline $\begin{array}{l}\text { Total THROAT score } \\
\text { (minimum, maximum) }\end{array}$ & $2(0,16)$ \\
\hline $\begin{array}{l}\text { Median pre-stroke mRS } \\
\text { (minimum, maximum) }\end{array}$ & $0(0,3)$ \\
\hline $\begin{array}{l}\text { Median mRS at recruitment } \\
\text { (minimum, maximum) }\end{array}$ & $4(2,5)$ \\
\hline \multicolumn{2}{|l|}{ Vascular risk factors } \\
\hline Hypertension & $21(72)$ \\
\hline Diabetes mellitus & $8(28)$ \\
\hline Previous stroke or TIA & $7(24)$ \\
\hline Coronary artery disease & $5(17)$ \\
\hline Atrial fibrillation & $9(31)$ \\
\hline Hyperlipidaemia & $16(55)$ \\
\hline Current smoker & $5(17)$ \\
\hline
\end{tabular}

NIHSS, indicates National Institutes of Health Stroke Scale score; OCSP, Oxfordshire Community Stroke Project; TACI, Total Anterior Circulation Infarction; PACI, Partial Anterior Circulation Infarction; POCI, Posterior Circulation Infarction; LACI, Lacunar Infarction; THROAT, The Holistic and Reliable Oral Assessment Tool; mRS, modified Rankin Scale; TIA, Transient Ischaemic Attack.

\section{Discussion}

Oral hygiene is a neglected and under-researched area of stroke care ${ }^{12,13}$, reflected by a lack of evidence-based guidelines. We developed an oral hygiene complex intervention for stroke unit care, combining staff education and training and an oral hygiene protocol comprising brushing with chlorhexidine gel and denture care. This intervention was feasible in an inpatient stroke service at a single UK centre, including both acute and rehabilitation stroke units.

Our staff training and education programme was informed by the training needs of frontline nursing staff and HCAs. It was intended to minimise the need for dental specialist input at the ward level, align with shift-work patterns and to be readily transferrable across sites for further study. The training and education content and format were both adequate and acceptable to the majority of staff, and its implementation entirely feasible across two ward areas. The oral hygiene protocol was safe, well-tolerated and acceptable in a sample of patients with a range of stroke severities and lengths of stay. Our patient sample was generally representative of UK stroke unit care ${ }^{29}$, although median NIHSS was higher than that of the national average. This implies that our oral hygiene protocol was feasible and well-tolerated even in patients with more severe stroke, where delivery of oral hygiene may be more challenging.

Poor oral health has several important implications and sequelae in patients hospitalised with stroke $^{12}$. It has been hypothesised that changes in the oral biofilm may contribute to the risk of pneumonia complicating stroke, particularly in patients with swallowing impairment. Indeed, dysphagia and aspiration are major risk factors for stroke-associated pneumonia (SAP) ${ }^{30}$. This is supported by a previous phase 2 trial which reported a reduction in SAP in stroke patients randomised to oral antibiotic gel, compared to placebo ${ }^{31}$. As SAP is associated with an increased risk of mortality, length of hospital stay and worse clinical outcomes in survivors ${ }^{32}$, oral hygiene may therefore be a therapeutic target for improving clinical outcomes in stroke care. However, brushing in the acute phase of stroke may be potentially harmful due to disruption of biofilm and enhanced risk of aspiration. We attempted to minimise this risk by utilising chlorhexidine gel or non-foaming toothpaste and allowing staff to use bedside suction at their discretion.

Several studies have evaluated oral hygiene interventions in hospitalised stroke patients. In a Korean study, patients with haemorrhagic or ischaemic stroke on an intensive care unit were randomised to either a chlorhexidine-based oral hygiene protocol administered by a dentist or usual care ${ }^{18}$. After an average of 2 weeks, there was a significant decrease in the plaque and 
gingival indices in the intervention group, although neither frequency of pneumonia nor clinical outcomes were presented. In a larger study of mechanically ventilated patients with brain injury $(30 \%$ with intracerebral haemorrhage), there was no difference in ventilator-associated pneumonia between groups randomised to either povidone-iodine naso- and oropharyngeal rinse or placebo ${ }^{19}$. A study on a stroke rehabilitation unit randomised patients to either professional oral hygiene instruction (OHI) by a dentist, $\mathrm{OHI}$ and chlorhexidine mouthrinse or OHI, chlorhexidine mouthrinse and twice-daily assisted brushing ${ }^{17}$. All participants were provided with a powered brush and nurses received lecture-based training sessions. Plaque and gingival bleeding indices were lower in the two chlorhexidine intervention groups. There were no episodes of pneumonia at all during the study period, which probably reflects the relatively low risk of pneumonia in the post-acute, rehabilitation phase. Brady et al. ${ }^{16}$ developed an oral hygiene complex intervention based on staff training and individualised oral healthcare assessment and care planning. Their intervention was feasible in a mixed methods study, although $50 \%$ of participants were discharged home within the first week, and chest infection occurred in only $2.5 \%$.

Our study has several limitations. Firstly, we evaluated the intervention within a single UK centre, which may have implications for generalisability. Although the staff education and training programme was feasible in an NHS setting, improvements could be made in the time taken for implementation in a multicentre trial setting. Refinements to hosting and accessing the webbased resource, embedding the programme within nursing staff mandatory training with oversight by a study coordinator may facilitate this. We used powered brushes as the default choice for our oral hygiene protocol, which has significant cost implications for roll-out in a multicentre study. Any potential benefits of powered brushing over manual brushes in this clinical setting, particularly with the proportion of edentulous patients, requires further study. We acknowledge the potential challenges in undertaking semistructured interviews in individuals with recent stroke. However, the interviews included carers where necessary and were conducted by a qualitative researcher with experience in interviewing older adults with language or communication problems. We did not use any detailed dental indices or scores for assessing effects of the intervention on plaque and gingivitis. However, an independent association between such measures in stroke patients with SAP or important clinical outcomes is yet to be established. We did not define SAP using rigorous diagnostic criteria. The variation in approach to diagnosis of SAP is wellrecognised $^{33}$, and clinicians may over-diagnose SAP compared to algorithm-based approaches ${ }^{34}$. In our study, the prevalence of lower respiratory tract infection was higher than that seen for unselected stroke admissions to our unit and also unselected admissions to stroke units nationally ${ }^{29}$. It was also higher than other studies of stroke unit oral hygiene interventions ${ }^{16,17}$. This is likely to relate to the differences in characteristics of patients (and their risk of developing pneumonia) and approaches used to diagnose pneumonia ${ }^{29,33}$. Consensus-based operational criteria for diagnosing SAP have recently been proposed, and such criteria could be used in future studies ${ }^{35}$.

\section{Conclusions}

The role of oral hygiene interventions in hospitalised stroke patients remains uncertain and requires further evaluation. Our oral hygiene complex intervention combining staff education and training, and an oral care protocol comprising brushing with chlorhexidine gel and denture care was feasible in a single UK stroke centre. Phase 2 multicentre studies optimising selection of the target population, modelling health economics and with carefully chosen validated outcomes are required.

\section{Acknowledgements}

This study was funded by the NIHR Collaboration in Leadership and Applied Health Research and Care (CLAHRC) for Greater Manchester. The views expressed in this article are those of the authors and not necessarily those of the NHS, NIHR or the Department of Health. The powered toothbrushes were kindly provided by Philips Oral HealthCare Ltd. We are very grateful to the SRFT Comprehensive Stroke Network Research Practitioners for their assistance with patient screening, recruitment and study conduct. We also gratefully acknowledge the staff at MANDCP (Manchester School for Dental Care Professionals) for their assistance developing the web-based training resource and training, the nursing staff and healthcare assistants at SRFT for participating in the staff training and focus groups and Dr Marko de Jager (Philips Research) for his helpful comments on the manuscript. 


\section{References}

1. http://www.who.int/mediacentre/factsheets/fs310/en/ (last accessed 09 December 2015).

2. http://www.who.int/healthinfo/global_burden_disease/GBD_report_2004update_full.pdf (last accessed 09 December 2015).

3. https://www.stroke.org.uk/sites/default/files/stroke_statistics_2015.pdf (last accessed 09 December 15).

4. Schillinger T, Kluger W, Exner M, Mlekusch W, Sabeti S, Amighi J et al. Dental and periodontal status and risk for progression of carotid atherosclerosis. The inflammation and carotid artery risk for atherosclerosis study dental substudy. Stroke 2006; 37: 2271-6.

5. Asai $K$, Yamori M, Yamazaki $T$, Yamaguchi A, Takahashi K, Sekine A et al. Tooth loss and atherosclerosis: the Nagahama study. J Dent Res 2015; 94 (3 Suppl): 52S-8S.

6. Lafon A, Pereira B, Dufour T, Rigouby V, Giroud M, Béjot $Y$ et al. Periodontal disease and stroke: a meta-analysis of cohort studies. Eur J Neurol 2014; 21: 1155-61.

7. Dai R, Lam OL, Lo EC, Li LS, Wen Y, McGrath C. A systematic review and meta-analysis of clinical, microbiological, and behavioural aspects of oral health among patients with stroke. J Dent 2015; 43: 171-80.

8. Azarpazhooh A, Leake JL. Systematic review of the association between respiratory diseases and oral health. J Periodontol 2006; 77: 1465-82.

9. Schimmel M, Leemann B, Christou $\mathbf{P}$, Kiliaridis S, Schnider A, Herrmann FR et al. Oral healthrelated quality of life in hospitalised stroke patients. Gerodontology 2011; 28: 3-11.

10. Sjögren $\mathbf{P}$, Nilsson E, Forsell $\mathbf{M}$, Johansson O, Hoogstraate J. A systematic review of the preventative effect of oral hygiene on pneumonia and respiratory tract infection in elderly people in hospitals and nursing homes: effect estimates and methodological quality of randomised controlled trials. $J$ Am Geriatr Soc 2008; 56: 2124-30.

11. Shi Z, Xie H, Wang P, Zhang $\mathbf{Q}$, Wu Y, Chen E et al. Oral hygiene care for critically ill patients to prevent ventilator-associated pneumonia. Cochrane Database Syst Rev 2013; 8: CD008367.

12. Kwok C, McIntyre A, Janzen S, Mays R, Teasell R. Oral care post stroke: a scoping review. J Oral Rehabil 2015; 42: 65-74.

13. Horne M, McCracken G, Walls A, Tyrrell PJ, Smith CJ. Organisation, practice and experiences of mouth hygiene in stroke unit care: a mixed methods study. J Clin Nurs 2014; 24: 728-38.

14. Talbot A, Brady M, Furlanetto DL, Frenkel H, Williams BO. Oral care and stroke units. Gerodontology 2005; 22: 77-83.

15. Fields LB. Oral care intervention to reduce incidence of ventilator-associated pneumonia in the neurologic intensive care unit. $J$ Neurosci Nurs 2008; 40: 291-8.

16. Brady MC, Stott DJ, Norrie J, Chalmers C, St George B, Sweeney PM et al. Developing and evaluating the implementation of a complex intervention: using mixed methods to inform the design of a randomised controlled trial of an oral healthcare intervention after stroke. Trials 2011; 12: 168.

17. Lam OL, McMillan AS, Samaranayake LP, Li LS, MCGrath C. Randomized clinical trial of oral health promotion interventions among patients following stroke. Arch Phys Med Rehabil 2013; 94: 435-43.

18. Kim EK, Jang SH, Choi YH, Lee KS, Kim YJ, Kim SH et al. Effect of an oral hygienic care program for stroke patients in the intensive care unit. Yonsei Med J 2014; 55: 240-6.

19. Seguin P, Laviolle B, DahyotFizelier C, Dumont R, Veber B, Gergaud $\mathbf{S}$ et al. Effect of oropharyngeal povidone-iodine preventive oral care on ventilator-associated pneumonia in severely brain-injured or cerebral hemorrhage patients: a multicenter, randomized controlled trial. Crit Care Med 2014; 42: 1-8.

20. Teddlie C, Tashakkori A. Foundations of mixed methods research: integrating quantitative and qualitative approaches in the social and behavioral sciences. Thousand Oaks, CA: Sage Publications Inc, 2009.

21. Möhler R, Köpke S, Meyer G. Criteria for Reporting the Development and Evaluation of Complex Interventions in healthcare: revised guideline (CReDECI 2). Trials 2015; 16: 204.

22. Craig $\mathbf{P}$, Dieppe $\mathbf{P}$, Macintyre $\mathbf{S}$, Michie S, Nazareth I, Petticrew M; Medical Research Council Guidance. Developing and evaluating complex interventions: the new Medical Research Council guidance. BMJ 2008; 337: al 655 .
23. Brady M, Furlanetto D, Hunter RV, Lewis S, Milne V. Staff-led interventions for improving oral hygiene in patients following stroke. Cochrane Database Syst Rev 2006; Art. No.: CD003864.

24. Yaacob M, Worthington HV, Deacon SA, Deery C, Walmsley AD, Robinson PG et al. Powered versus manual toothbrushing for oral health. Cochrane Database Syst Rev 2014; Issue 6. Art. No.: CD002281.

25. Paraskevas S, Rosema NA, Versteeg $P$, Timmerman MF, van der Velden $U$, van der Weijden GA. The additional effect of a dentifrice on the instant efficacy of toothbrushing: a crossover study. $J$ Periodontol 2007; 78: 1011-6.

26. Frenkel HF, Harvey I, Newcombe RG. Improving oral health in institutionalised elderly people by educating caregivers: a randomised controlled trial. Community Dent Oral Epidemiol 2001; 29: 289-97.

27. Dickinson H, Watkins $C$, Leathley $\mathbf{M}$. The development of the THROAT: the holistic and reliable oral assessment tool. Clin Eff Nurs 2001; 5: 104-10.

28. Ritchie J, Spencer L, O'Connor W. Carrying out qualitative analysis. In: Ritchie J, Lewis J eds. Qualitative Research Practice: A Guide for Social Science Students and Researchers. Thousand Oaks, CA: Sage, London, 2003: 219-62.

29. Smith CJ, Bray BD, Hoffman A, Meisel A, Heuschmann PU, Wolfe CDA et al. Can a novel clinical risk score improve pneumonia prediction in acute stroke care? A UK multicenter cohort study J Am Heart Assoc 2015; 4: e001307.

30. Martino R, Foley N, Bhogal S, Diamant N, Speechley $M$, Teasell R. Dysphagia after stroke: incidence, diagnosis, and pulmonary complications. Stroke 2005; 36: 2756-63.

31. Gosney M, Martin MV, Wright AE. The role of selective decontamination of the digestive tract in acute stroke. Age Ageing 2006; 35: 42-7.

32. Finlayson O, Kapral $\mathbf{M}$, Hall $\mathbf{R}$, Asllani E, Selchen D, Saposnik G; Canadian Stroke Network; Stroke Outcome Research Canada (SORCan) Working Group. Risk factors, inpatient care, and outcomes of pneumonia after ischemic stroke. Neurology 2011; 77: 1338-45.

33. Kishore AK, Vail A, Chamorro A, Garau J, Hopkins SJ, Di Napoli $\mathbf{M}$ et al. How is pneumonia 
diagnosed in clinical stroke research? A systematic review and meta-analysis Stroke 2015; 46: 1202-9.

34. Kalra L, Irshad S, Hodsoll J, Simpson $M$, Gulliford $M$, Smithard $D$ et al. Prophylactic antibiotics after stroke for reducing pneumonia in patients with dysphagia (STROKEINF): a prospective, cluster-randomised, open-label, masked endpoint, controlled clinical trial. Lancet 2015; 386: 1835-44.

35. Smith CJ, Kishore AK, Vail A, Chamorro A, Garau J, Hopkins SJ et al. Diagnosis of stroke-associated pneumonia: recommendations from the pneumonia in stroke con- sensus group. Stroke 2015; 46: 2335-40.

\section{Supporting Information}

Additional Supporting Information may be found in the online version of this article:

Table S1. Themes arising from focus groups after implementation of the staff education and training programme.

Table S2. Characteristics of participating stroke patients interviewed.
Table S3. Patients and carer perspectives.

Correspondence to:

Dr Craig J. Smith, Manchester Academic Health Science Centre, Salford Royal NHS

Foundation Trust, Room C238, Clinical Sciences Building, Salford, UK.

Tel.: +44 161 2060623

E-mail: Craig.Smith-2@ manchester.ac.uk 